\title{
Evolution of complex asexual reproductive strategies in jellyfish
}

\author{
Schnedler-Meyer, Nicolas Azaña; Pigolotti, Simone; Mariani, Patrizio
}

\section{Published in:}

American Naturalist

Link to article, DOI:

$10.1086 / 697538$

Publication date:

2018

Document Version

Publisher's PDF, also known as Version of record

Link back to DTU Orbit

Citation (APA):

Schnedler-Meyer, N. A., Pigolotti, S., \& Mariani, P. (2018). Evolution of complex asexual reproductive strategies in jellyfish. American Naturalist, 192(1), 72-80. https://doi.org/10.1086/697538

\section{General rights}

Copyright and moral rights for the publications made accessible in the public portal are retained by the authors and/or other copyright owners and it is a condition of accessing publications that users recognise and abide by the legal requirements associated with these rights.

- Users may download and print one copy of any publication from the public portal for the purpose of private study or research.

- You may not further distribute the material or use it for any profit-making activity or commercial gain

- You may freely distribute the URL identifying the publication in the public portal

If you believe that this document breaches copyright please contact us providing details, and we will remove access to the work immediately and investigate your claim. 


\title{
Evolution of Complex Asexual Reproductive Strategies in Jellyfish
}

\author{
Nicolas Azaña Schnedler-Meyer, ${ }^{1, *}$ Simone Pigolotti, ${ }^{2}$ and Patrizio Mariani ${ }^{1}$ \\ 1. Centre for Ocean Life, National Institute of Aquatic Resources, Technical University of Denmark Kemitorvet, Building 202, 2800 Kgs. \\ Lyngby, Denmark; 2. Okinawa Institute of Science and Technology 1919-1 Tancha, Onna-son, Kunigami-gun Okinawa, Japan 904-0495 \\ Submitted August 31, 2017; Accepted January 31, 2018; Electronically published April 30, 2018 \\ Online enhancements: appendix. Dryad data: http://dx.doi.org/10.5061/dryad.506g4.
}

\begin{abstract}
AвSTRACT: Many living organisms in terrestrial and aquatic ecosystems rely on multiple reproductive strategies to reduce the risk of extinction in variable environments. Examples are provided by the polyp stage of several bloom-forming jellyfish species, which can reproduce asexually using different budding strategies. These strategies broadly fall into three categories: (1) fast localized reproduction, (2) dormant cysts, or (3) motile and dispersing buds. Similar functional strategies are also present in other groups of species. However, mechanisms leading to the evolution of this rich reproductive diversity are yet to be clarified. Here we model how risk of local population extinction and differential fitness of alternative modes of asexual reproduction could drive the evolution of multiple reproductive modes as seen in jellyfish polyps. Depending on environmental parameters, we find that evolution leads to a unique evolutionarily stable strategy, wherein multiple reproductive strategies generally coexist. As the extinction risk increases, this strategy shifts from a pure budding mode to a dual strategy and finally to one characterized by allocation into all three modes. We identify relative fitness-dependent thresholds in extinction risk where these transitions can occur and discuss our predictions in light of observations on polyp reproduction in laboratory and natural systems.
\end{abstract}

Keywords: evolutionarily stable state, life cycle, dormancy, dispersal, jellyfish polyps, evolutionary model.

\section{Introduction}

All ecosystems are subject to changes in environmental conditions, such as repeated natural cycles, episodic events, and long-term trends including climate change. To cope with such spatial and temporal variability, many organisms have evolved strategies that trade off their short-term average fitness in favor of a reduction of risk. These bet-hedging strategies maximize the geometric mean fitness, typically at the expense of a decreasing arithmetic mean fitness (Cohen 1966; Kussell and Leibler 2005; Childs et al. 2010). Bet-

\footnotetext{
* Corresponding author; email: niaz@aqua.dtu.dk. ORCIDs: Schnedler-Meyer, http://orcid.org/0000-0002-9725-9646.
}

Am. Nat. 2018. Vol. 192, pp. 72-80. (C) 2018 by The University of Chicago. 0003-0147/2018/19201-57916\$15.00. All rights reserved.

DOI: $10.1086 / 697538$ hedging strategies can be evolutionarily stable strategies (ESSs) and hence noninvasible by rare mutants, because the long-term evolutionary success of a species, genotype, or allele is sensitive to rare occurrences of very low fitness or extinction (see, e.g., Comins et al. 1980). In multicellular organisms, a classic example of bet hedging is the evolution of delayed germination (dormancy) or mechanisms of dispersal in the seeds of annual plants (Comins et al. 1980; Venable and Brown 1988; Snyder 2006; Vitalis et al. 2013). Delayed germination and increased dispersal cause the seeds of a single generation to germinate in different years or at different locations, respectively, spreading the risk of low fitness due to adverse conditions. These strategies come at the cost of a reduced fitness in good conditions, because a fraction of propagules do not germinate- or are exported out of the favorable areas - and because of costs associated with the mechanisms themselves.

In general, the complex interplay between environment and life-history traits generates the variety of strategies observed in living organisms. An emblematic example is provided by scyphozoan jellyfish. Widely successful and conspicuous, scyphozoans have attracted much attention in recent decades, due to the ability of many species to form large, irregular blooms of their sexually reproducing adults (medusae), with often drastic consequences for ecosystems and human activities (Condon et al. 2012; Graham et al. 2014). The large and conspicuous medusae are produced in large numbers by small, inconspicuous, and sessile polyps, which are themselves usually capable of multiplying through various modes of asexual budding. Therefore, polyp population size ultimately determines the biomass of adult medusae (Hamner and Dawson 2009; Lucas et al. 2012; Schiariti et al. 2014). In recent years, experimental evidence has led to increased knowledge of the polyp propagation strategies available to different species and to the environmental conditions that trigger their expression (Adler and Jarms 2009; Lucas et al. 2012; Schiariti et al. 2014, 2015). Reproductive strategy in scyphozoan polyps is extremely varied: some species have access to a variety of asexual reproductive modes, 
while others employ only one or two. It is still unclear exactly what drives this diversity in reproductive strategy and how and under which conditions it contributes to maintaining polyp populations. Though hypotheses have been put forward about the trade-offs between the various strategies in multimodal species (Schiariti et al. 2014), we are not aware of an explicit analysis of why similar species of polyps have evolved different reproductive strategies.

Asexual reproduction by scyphozoan polyps broadly fall into three general modes, which can be present in the same individual (Vagelli 2007; Adler and Jarms 2009; see fig. 1, top): (1) The production of local clones of the parent polyp through budding (hereafter, local budding). The new clone is usually able to start feeding before completion and detachment, making this a very fast reproductive mode under favorable conditions. (2) The production of resting cysts, which are resistant to starvation, predation, and other adverse conditions (up to periods of several years). Cysts require a specific set of environmental conditions to trigger germination, making them the slowest reproductive strategy.

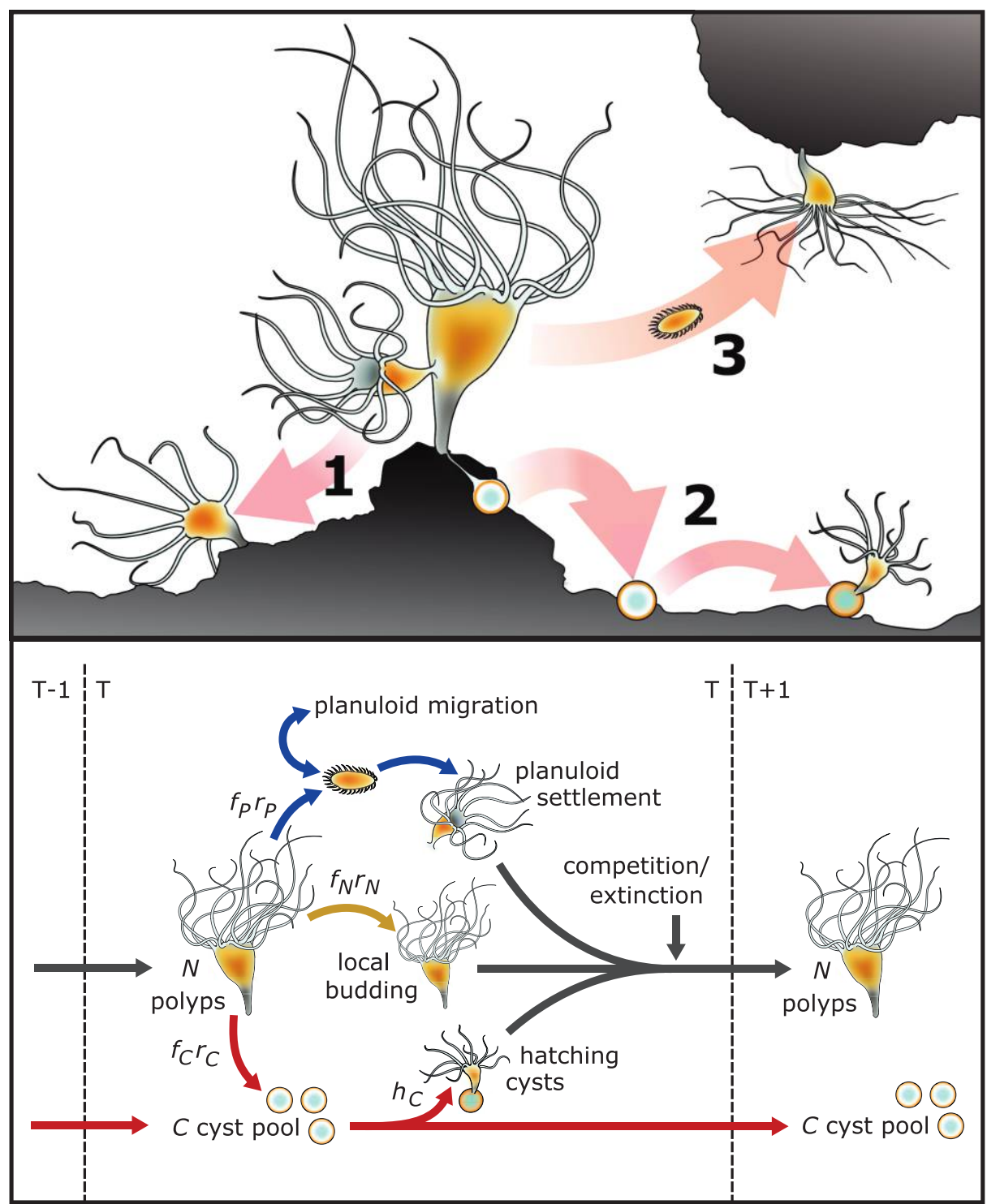

Figure 1: Reproductive strategy and model structure. Top, asexual reproductive modes in scyphozoan polyps: (1) The budding off of local buds, which are more or less fully developed before detachment. (2) The production of cysts, which are dormant until they hatch into a new polyp at a later time (months-years). (3) The production of motile buds of various types, which go through a dispersing phase before attachment and development. Bottom, illustration of the model structure in a single patch, where $N$ and $C$ are the numbers of polyps and cysts, respectively, at the start of the time step; $f$ denotes the fraction of reproductive effort allocated to a given mode; $r$ is the relative fitness of a given reproductive mode; and $h_{\mathrm{C}}$ is the hatching rate of cysts. 
(3) The production of (generally smaller) motile and dispersing buds that do not settle and develop into polyps until after a period of time (days to weeks).

In both terrestrial and aquatic ecosystems, organisms that can reproduce in several ways often employ a fast, local asexual reproductive mode in addition to sexual reproduction through smaller, dispersing, or dormant propagules. The underlying trade-off has been studied in the context of sexual versus asexual reproduction (see, e.g., Hidalgo et al. 2016) and dispersal versus vegetative propagation (Harada and Iwasa 1994). It is of general interest to disentangle the complex trade-offs between dispersal, dormancy, and fast local reproduction strategies. In this broader perspective, scyphozoan polyps represent a highly suitable study system, because of the large variation within the group. Moreover, the three reproductive modes are all asexual and independent in polyps, rather than being traits of the same propagule, as in plant seeds.

In this article, we model the optimal allocation of scyphozoan polyps to the three asexual reproductive modes under varying parameters, characterizing environmental conditions. To this end, we introduce a simple evolutionary model, analyzing the emergence of evolutionarily stable strategies in the trait space of allocation among the strategies of dispersal, dormancy, and rapid local reproduction in a densitydependent metapopulation, evolving on a spatial lattice. We investigate whether a single optimal strategy exists for a given set of conditions, and under which conditions we can expect a mix of the three reproductive modes. We conclude by contrasting our results with the literature on scyphozoan polyp reproduction and propose further experiments on the evolution of reproduction in scyphozoan polyps.

\section{Methods \\ Competition Model}

We introduce a metapopulation model describing two competing genotypes of a scyphozoan polyp species: a wild type and a mutant. The two genotypes occupy an $L$-by- $L$ square lattice of patches, each representing a suitable local habitat.

In each patch $i$, the two competing genotypes can, in principle, coexist. The local fractions of polyps at generation $t$ belonging to the two types are $N_{i . t}^{w}$ and $N_{i . t}^{m}$. In the first generation $(t=1)$, the mutant and wild type are present in equal fractions in each patch $\left(N_{i .1}^{w}=N_{i .1}^{m}=0.5\right.$ for all $\left.i\right)$. The individual polyps have access to each of the three reproductive modes of local polyps, motile buds, and cysts, but the two genotypes differ in their relative allocation. The relative allocation to local buds we call $f_{\mathrm{B}}$, to motile buds $f_{\mathrm{M}}$, and to cysts $f_{\mathrm{C}}$, so that, for the wild type,

$$
f_{\mathrm{B}}^{w}+f_{\mathrm{M}}^{w}+f_{\mathrm{C}}^{w}=1,
$$

while the same applies for mutants. Apart from this difference in reproductive allocation, the two genotypes are equal. We assume that the growth rates are sufficiently large, so that at the end of each generation each patch is either saturated or empty (in the case of a local extinction event, as discussed later). The population density of a saturated patch is conventionally set to 1 . At each generation and patch, we compute the production of propagules of each of the three types for the two genotypes (fig. 1), such that (1) newborn local buds are simply retained in their local patch and added to the local population of their respective genotype and (2) motile buds are also immediately added to polyp populations, but the production of each individual patch is evenly distributed among itself and the neighboring eight patches. We assume periodic boundary conditions to avoid border effects. (3) Cysts are retained in their local patch but do not immediately contribute to the polyp population. Instead, they enter the local cyst pool, a fraction $h_{\mathrm{C}}$ of which hatches in each generation.

The number of newborns from each mode depends on their relative fitness. We fix the reproductive success of local budding to a value of 1 but vary those of the other two strategies $\left(r_{\mathrm{M}}\right.$ for motile buds and $r_{\mathrm{C}}$ for cysts) relative to this, such that the numbers of newborns in patch $i$ at generation $t$ by local budding, motile bud immigration, and cyst hatching are for the wild type

$$
\begin{aligned}
B_{i . t}^{w} & =N_{i . t-1}^{w} f_{\mathrm{B}}^{w}, \\
M_{i . t}^{w} & =\sum_{j=1}^{9}\left(\frac{1}{9} N_{j . t-1}^{w} f_{\mathrm{M}}^{w} r_{\mathrm{M}}\right), \\
H_{i . t}^{w} & =C_{i . t-1}^{w} h_{\mathrm{C}},
\end{aligned}
$$

respectively, while the same applies for mutants. Here, $j$ is one of the nine patches (including itself) from which patch $i$ receives immigrants, and $C_{i . t-1}$ is the number of cysts in the cyst pool of patch $i$ at time $t-1$. The cyst pool increases by the production of cysts and decreases as cysts hatch, according to

$$
C_{i . t}^{w}=C_{i . t-1}^{w}\left(1-h_{\mathrm{C}}\right)+N_{i . t-1}^{w} f_{\mathrm{C}}^{w} r_{\mathrm{C}} .
$$

In each generation, the contribution of new cysts to the cyst pool is calculated after the number of hatching cysts to prevent cysts from hatching in the same generation in which they are produced.

The newborns from each reproductive mode are then added together to form the total number of newborn polyps $S_{i . t}^{w}$ and $S_{i . t}^{m}$ in patch $i$ and generation $t$. Local competition is implemented by normalizing the final production of polyps of both genotypes, such that $N_{i . t}^{w}+N_{i . t}^{m}=1$ :

$$
\begin{aligned}
N_{i . t}^{w} & =\frac{S_{i . t}^{w}}{S_{i . t}^{w}+S_{i . t}^{m}}, \\
N_{i . t}^{m} & =\frac{S_{i . t}^{m}}{S_{i . t}^{w}+S_{i . t}^{m}} .
\end{aligned}
$$


Finally, at the end of each generation, local extinction can randomly and independently occur with probability $e$ in each patch, so that the resulting number of extinction is binomially distributed. In such a case, all polyps of both types are killed; that is, both $N_{i . t}^{w t}$ and $N_{i . t}^{m}$ are set to 0 . An empty patch can be recolonized from the cyst bank or through immigration of motile buds at a later time. In this initial version of the model, local extinction events are independent among different patches. We also investigate the case where extinctions are correlated in space, that is, when extinction events span a larger area than a single patch. The size of these areas is characterized by the spatial correlation length $r$. In this variant of the model, a fixed proportion $e$ of patches undergoes extinction every generation. See appendix, available online, for a detailed description of the algorithm and examples of the spatial patterns of extinctions.

\section{Evolutionary Model}

Our aim is to compare different reproductive strategies, characterized by their relative allocation to local buds, motile buds, and cysts and to identify possible evolutionarily stable strategies (ESSs). ESSs are characterized by not being invasible by a small population of mutants employing any different strategy. To compute ESSs, we use an evolutionary scheme based on random mutation and selection. Starting from a given wild type, we generate a mutation, changing the allocation to reproduction in a random direction such that

$$
\delta_{\mathrm{F}}=\sqrt{\Delta f_{\mathrm{B}}^{2}+\Delta f_{\mathrm{M}}^{2}+\Delta f_{\mathrm{C}}^{2}}
$$

where $\delta_{\mathrm{F}}$ is the distance between the mutant and the wild type in allocation space. The competition model can then be simulated until either the wild type or the mutant reach global extinction.

The surviving genotype becomes the wild type for the next iteration. These dynamics ensure evolution of the wild type toward increased competitive ability. We terminate the evolutionary dynamics once we find a wild type that has not been outcompeted for a number $G_{\max }=40$ of consecutive iterations, and we approximate the ESS with such a wild type.

The model code is written in MATLAB and is open access; it is available through the Dryad Digital Repository: https://dx.doi.org/10.5061/dryad.506g4 (Schnedler-Meyer et al. 2018).

\section{Choice of Life Stages}

The goal of this study is limited to comparing the different types of asexual reproduction available to polyps rather than modeling the evolution of the entire reproductive cycle in jellyfish. To this end, our model is simplified and does not describe the adult medusa stage of jellyfish. For our aims, an interesting question is to which extent the presence of medusae affect the trade-off between the three reproductive modes of polyps. In principle, medusa dispersal and reproduction could alter this balance, reducing the benefit of recolonization of new habitats and increasing competition for space. Ultimately, however, we expect that including medusae will affect our results quantitatively but not qualitatively, and at this stage including them in the model would complicate the interpretation of the predictions about polyps without adding significant ecological insight. We note that while medusae cannot reproduce without going through the polyp stage, polyps can reproduce without undergoing the medusa stage and can therefore be considered independent to some extent. Additionally, from the polyp perspective, the main relevance of the medusa stage is to maintain the quality of the gene pool via sexual reproduction, an aspect that is out of the scope of our study but is partially incorporated in the mutation rate of the evolutionary model.

\section{Results}

Simulations of the competition model always tend toward a state where one of the two genotypes, depending on parameter choices, outcompetes the other (fig. $2 A$ ). The difference between growth rates of the two genotypes also influenced the number of generations needed to reach monodominance.

Letting the wild type evolve according to the evolutionary model, we observed convergence to an ESS characterized by distinct allocations to the three strategies $f_{\mathrm{B}}, f_{\mathrm{M}}$, and $f_{\mathrm{C}}$ (fig. $2 B$ ). Importantly, given a fixed extinction risk $e$ and relative fitness $r_{\mathrm{M}}$ and $r_{\mathrm{C}}$, the evolutionary model always converged to the same strategy, independently from the initial distribution of wild and mutant types. This is represented in figure $2 C$, where strategies are represented as points in relative allocation space, that is, the triangular surface where equation (1) is satisfied and all relative allocations are between 0 and 1 . This indicates that, for a given set of parameters, the ESS is unique and independent of initial conditions. Notice that the evolutionary trajectories show some degree of stochasticity due to the randomness of mutations (fig. 2C). Nevertheless, the tracks tend to move in the direction of highest local fitness, thus sketching the portrait of the fitness landscape. For example, when $r_{\mathrm{M}}$ and $r_{\mathrm{C}}$ are similar, the population tends to converge toward the optimal allocation to local buds $\left(f_{\mathrm{B}}\right)$ and then motile buds $\left(f_{\mathrm{M}}\right)$ and cysts $\left(f_{\mathrm{C}}\right.$; see fig. $2 C$ ). This indicates a higher selection pressure on the fraction of local buds and a shallower fitness gradient between the fractions of motile buds and cysts. However, when $r_{\mathrm{M}}$ and $r_{\mathrm{C}}$ are different, evolutionary trajectories tend more 

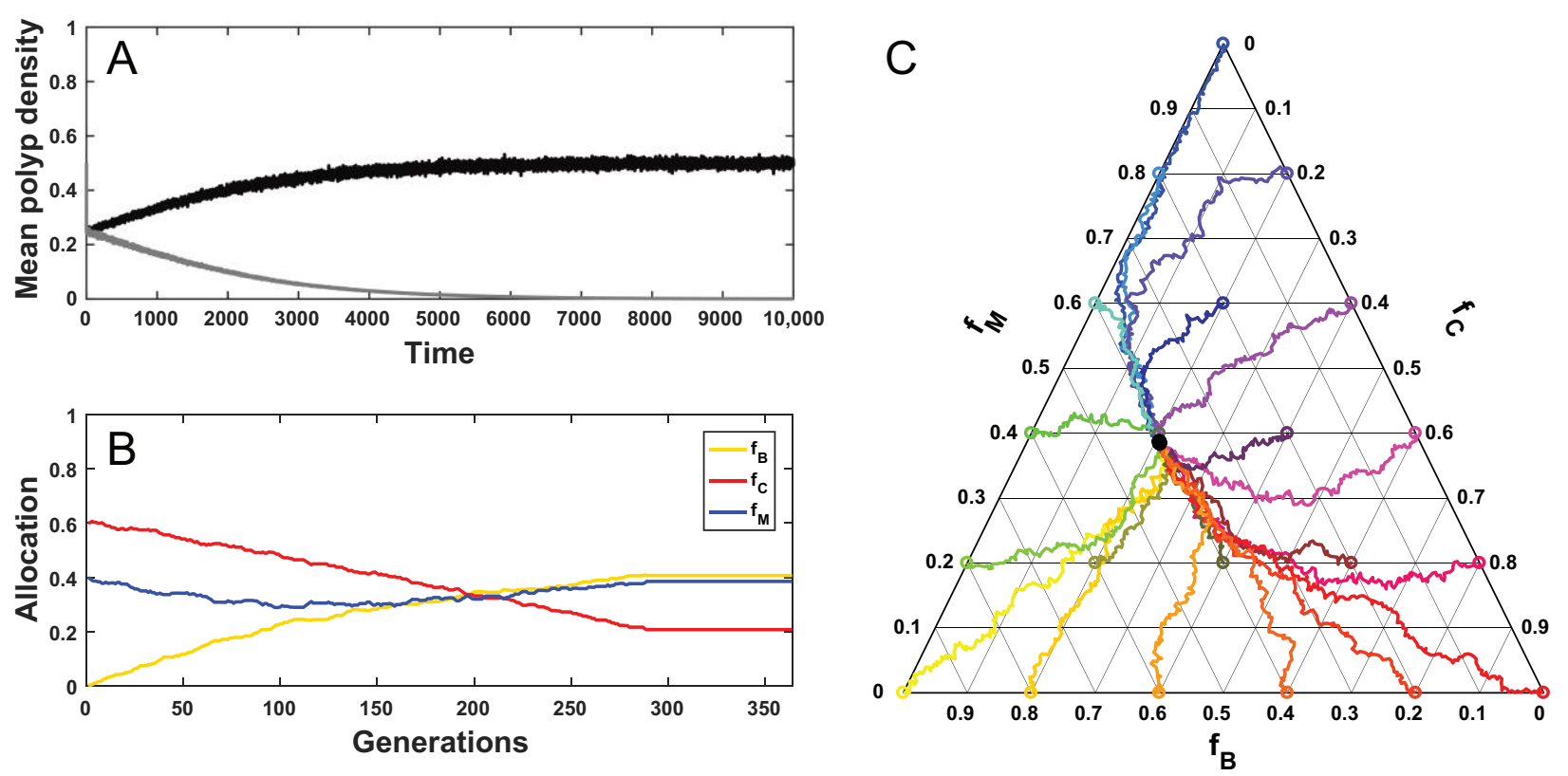

Figure 2: Evolution of strategies in the model. $a$, Competition between a wild type ( $w$, gray) and a mutant ( $m$, black) polyp, leading to competitive exclusion of the wild type by the mutant. $b$, Evolution of allocation to the three reproductive modes through generations of mutation and selection. After $\sim 290$ generations, the system has reached an evolutionarily stable strategy (ESS). $c$, Convergent evolution to a common ESS of populations starting with different initial allocation values. For all simulations, parameters are $L=50$, both $r_{\mathrm{M}}$ and $r_{\mathrm{C}}=0.25$, and $e=0.5$. The fractions of reproductive resources allocated to motile buds, cysts, and local budding are indicated by $f_{\mathrm{M}}, f_{\mathrm{C}}$, and $f_{\mathrm{B}}$, respectively.

directly toward the ESS, indicating a more pronounced fitness gradient.

Repeating this analysis for different parameter values, we found that both the fitness landscape and the corresponding ESS can change. In particular, the strategy allocation at the ESS is regulated by the extinction risk and the relative values of $r_{\mathrm{M}}$ and $r_{\mathrm{C}}$ (fig. $3 A$ ). At low extinction risk, local budding is favored, and the ESS is dominated by this strategy at vanishing extinction risk; that is, $f_{\mathrm{B}} \rightarrow 1$, for $e \rightarrow 0$. Increasing $e$, allocation to local budding decreases approximately linearly, and the optimal allocation shifts toward production of cysts and/or motile buds, with $f_{\mathrm{B}} \rightarrow 0$, when $e \rightarrow 1$. At low or intermediate extinction risk, the ESS is dominated by two modes: local budding and either cysts or motile bud production, except for when their production rates are roughly equal (fig. $3 A$ ). At higher extinction risk, all three reproductive modes do contribute to the ESS, which moves toward relatively similar investment into motile buds and cysts, even when their relative fitness is very different. Finally, at extremely high extinction risks $(>0.9)$, the ESS tends to a pure cyst strategy, except when $r_{\mathrm{C}}$ is 0 .

Introducing spatial autocorrelation to the extinction rate changes the optimal allocation of resources between cysts and motile buds significantly (fig. $3 B$ ). The general shape of the curve is conserved, confirming the seemingly strong selective pressure toward a strategy characterized by all three reproductive modes at high extinction probabilities. However, the ESS is increasingly skewed in favor of cysts as the autocorrelation length increases (fig. $3 B$ ), indicating a reduced benefit of allocation to motile buds.

These results are qualitatively robust to changes in other parameters of the model. Similar ESS and evolutionary trajectories are observed at different sums of $r_{\mathrm{M}}+r_{\mathrm{C}}$ (see fig. A2A, A2B; figs. A1, A2 are available online).The main effect of increasing $r_{\mathrm{M}}+r_{\mathrm{C}}$ is to skew the general pattern in figure $3 A$ away from allocation to local budding. Increasing the hatching rate $h_{\mathrm{C}}$ has the same general effect as lowering $r_{\mathrm{C}}$, meaning that the cysts become more advantageous if they persist in the seed bank for a larger number of generations (fig. A2C). We verified that all these qualitative behaviors are insensitive to lattice size.

\section{Discussion}

\section{Evolution of Reproductive Strategies}

We modeled optimal allocation to different asexual reproduction modes in variable environments. The model describes the evolution of three distinct reproductive modes in jellyfish polyps: dispersal (motile buds), dormancy (cysts), and rapid local reproduction (local budding). These strategies are common among both terrestrial and aquatic organ- 
A

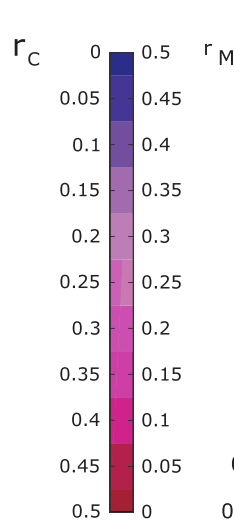

B

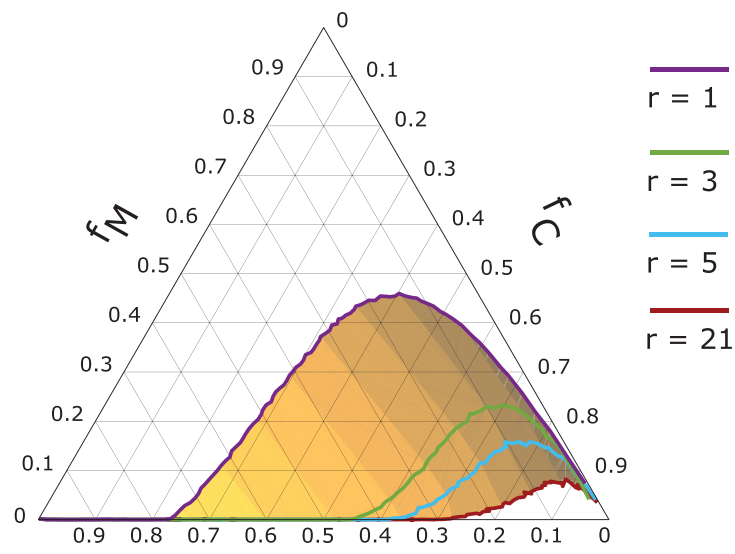

$f_{B}$

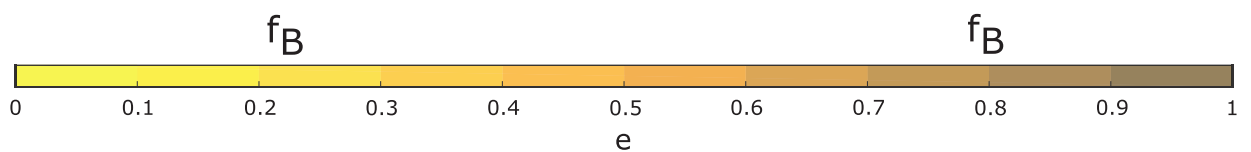

Figure 3: $A$, Evolutionarily stable strategy (ESS) for different parameter choices of relative fitness of motile buds and cysts $\left(r_{\mathrm{M}}\right.$ and $r_{\mathrm{C}}$, respectively) and extinction risk $e$. Lines show the location of the ESS along the gradient of $e$ (shading) for different sets of $r_{\mathrm{M}}$ and $r_{\mathrm{C}}$, the sum of which is kept constant at a value of $r_{\mathrm{M}}+r_{\mathrm{C}}=0.5$. B. As in $A$, but for different values of spatial correlation length $r$. All four lines are with $r_{\mathrm{M}}=0.25$ and $r_{\mathrm{C}}=0.25$. As spatial correlation increases (increasing $r$ ), the ESS moves toward increased allocation to cysts, as shown by the arrows, which are between points with equal extinction risk.

isms; hence the results of the model have implications beyond jellyfish ecology. The model predicts mixed evolutionarily stable reproductive strategies for a large range of parameter values, consistent with the presence of multiple reproductive modes in plants (e.g., Imbert 2002), invertebrates (e.g., Marcus 1996), and vertebrates (e.g., Touchon and Warkentin 2008). Indeed, we predict pure strategies only when the extinction risk $e$ is negligible (pure local budding strategy) or when it approaches the extreme case $e=1$ (pure cyst strategy). This result can be understood in a bethedging framework. Although local rapid reproduction is always superior in a risk-free environment, strategies that have lower instantaneous fitness but decrease the impact of local extinctions can successfully invade a risky environment. In our particular case, motile buds and cysts can reduce the risk of local extinction by spreading the offspring in space and time, at the cost of a lower relative fitness of cysts and motile buds compared to local budding. Generally, we find that local extinction risk is the main determinant of the evolution of the different strategies. At low-to-intermediate extinction risk, dispersal and dormancy modes are somewhat complementary and coexist only when their relative fitness is similar. However, when $e$ becomes intermediate to high, mixed strategies are selected, even when one mode has a much higher relative fitness, because cysts and motile polyps are exposed to different risks. Cysts are vulnerable when the same cell undergoes extinction for several consecutive generations, while motile buds are vulnerable when several adjacent cells go extinct in a single generation. As $e$ becomes higher and both kinds of events become common, the population must defend against both risks.

These results can be compared with studies on plants, where dormancy and dispersal of seeds are likewise predicted to increase as the local extinction rate increases (e.g., Comins et al. 1980; Venable and Brown 1988; Vitalis et al. 2013; but see also Ronce et al. 2000). In most plants, dormancy and dispersal are properties of the same seed, where they may interfere with each other or impose different requirements on seed traits (Vitalis et al. 2013). Many theoretical studies suggest that dormancy and dispersal should evolve to be negatively correlated, consistent with our results at low extinction risk (Levin et al. 1984; Cohen and Levin 1987; Venable and Brown 1988; Kobayashi and Yamamura 2000). Indeed, positive correlations emerge only in such systems when the environment is positively autocorrelated in time, where dormancy counteracts the tendency of dispersing seeds to germinate in poor habitats (Cohen and Levin 1991; Snyder 2006). In our case, the mechanisms of dispersal and dormancy are tied to separate propagules and interact only through the allocation tradeoff. We observe that this can also lead to positively correlated dispersal and dormancy, even in a temporally uncor- 
related environment, depending on the level of extinction risk. Interestingly, our case is similar to a specific case in Vitalis et al. (2013), where plant dispersal and dormancy was found to be coselected when dormancy was allowed to evolve exclusively in philopatric seeds. Thus, the specific mechanisms of dormancy and dispersal are important for their trade-offs and interactions, and in particular, independent functioning of the two facilitates their coevolution.

The differing functioning of dispersal and dormancy is evident in the case of spatially autocorrelated extinctions. The heavy favoring of cysts in this case is linked to the limited dispersal distance (one cell) of motile buds, since in this case neighboring cells are likely to share the same condition as the focal cell. However, a less pronounced selection against motile buds can be expected if they can disperse further.

\section{Reproduction in Jellyfish Polyps}

Consistent with our prediction, coexistence of multiple asexual propagation modes in the polyps is widespread among scyphozoans (reviewed by Adler and Jarms 2009). Indeed, many species even have access to several distinct varieties of the three main reproductive modes considered in this study (Vagelli 2007; Schiariti et al. 2014). Although polyps play a large role in jellyfish bloom formation (Lucas et al. 2012), due to their cryptic nature, we do not yet have a clear overview of the distribution and expression of their reproductive modes. However, it is well known that examples of the three main modes considered here are found in all the major branches of Scyphozoa (Adler and Jarms 2009).

In nature, many scyphozoan species have access to local budding (Adler and Jarms 2009; Arai 2009), but to the best of our knowledge, there are no species that employ local budding only. This is consistent with the model outcome that a pure local budding strategy is evolutionarily stable only in the absence of an extinction risk, a condition that is unrealistic in natural environments. Further supporting this idea, species with access to local budding were shown to have much higher reproductive rates compared to those without in experiments where mortality is low and polyps are fed ad lib. (Schiariti et al. 2014). Under such conditions, both our results and experimental evidence suggest that species with access to several reproductive modes allocate 95\%$100 \%$ of their resources to local budding (Schiariti et al. 2014).

According to our model, investment into two or more reproductive modes is a signature of risky environments. High local extinction risk is common among polyp populations, as they typically occupy habitats characterized by frequent disturbances and intense competition for space (Clark 1975). Moreover, common predators of jellyfish polyps, like nudibranch mollusks, have high efficiency but low mobility, such that they are patchily distributed and tend to completely clear an area of polyps (Hoover et al. 2012; Takao et al. 2014). For example, the genus Aurelia, arguably the most successful jellyfish in the world, often inhabits variable and changeable estuarine and shallow environments (Lucas and Williams 1994; Lucas 2001; Møller and Riisgård 2007) and commonly employs all three reproductive modes.

Motile buds are perhaps the least frequent reproductive mode among scyphozoan jellyfish (Adler and Jarms 2009), although their presence might be underrepresented in the literature because they are even more cryptic than cysts and local buds. In the laboratory, both relative and absolute production of motile buds increases in response to high polyp densities (Schiariti et al. 2015). In patchy natural environments with common but spatially uncorrelated extinctions, occupied and empty habitats will be in close proximity, with significant potential for colonization, supporting long-term survival of the metapopulation. We predict that motile buds can have an important function in such habitats and should therefore be common - a prediction that can be tested in natural environments.

Cyst production seems to be the most ubiquitous reproductive mode, being present in the majority of species (Adler and Jarms 2009). In agreement with both intuition and our findings, cysts are usually produced in response to predation or starvation by species with access to several strategies, although species with access to only cyst production do increase it in response to favorable conditions (Arai 2009; Lucas et al. 2012; Schiariti et al. 2014). As discussed, cysts and motile buds differ in the type of environmental variation that they most efficiently counter. Initially, we assumed in the model that extinctions occur randomly and independently in space and time. However, when extinctions are correlated in space in a range larger than the dispersal distance of motile buds, the ESS moves toward increased allocation to cysts (fig. $3 B$ ). Examples of natural events causing such large-scale risks can be seasonal starvations and large fluctuations in temperature or salinity. Such events are common in natural (especially seasonal) environments, consistent with the prevalence of cysts among scyphozoan jellyfish.

Here, we considered evolutionary timescales and, for simplicity, assumed that patches are always either full or empty. Neglecting explicit dynamics within a patch is acceptable when the polyps reproduce very quickly, as in the case of scyphozoan polyps (Schiariti et al. 2015). However, in nature, the expression of the available reproductive modes by scyphozoan polyps can be adjusted in response to, for example, seasonal changes. Our model could be generalized to explicitly describe short-term population dynamics, although we do not expect significant qualitative differences in the optimal allocation of reproductive strategies, even when environmental fluctuations occur on shorter timescales. 


\section{Future Perspectives}

In addition to asexual reproduction, polyp populations in the wild are also at least occasionally reseeded by planula larvae produced by the sexual medusa phase (Lucas et al. 2012). While we did not include the adult generation in our model, we expect that a large and frequent influx of planulae will decrease the advantages of local budding and especially of motile buds, whose primary advantage is the reseeding of empty patches of substrate. In seasonal environments, where the growth rate of polyps can be low and polyps primarily serve as an overwintering stage, this might in part explain why cysts seem to be the most widespread reproductive strategy among scyphozoans (Adler and Jarms 2009). Polyp dynamics are often neglected in ecological models of jellyfish (but see, e.g., Ruiz et al. 2012), in spite of the increasing awareness of the importance of polyps for jellyfish population dynamics. An evolutionary model combining detailed descriptions of both the polyp and medusa stages of jellyfish would certainly be an interesting future perspective. However, the complexity of the ecological and spatial dynamics that must be included in such a model, combined with the relatively limited empirical evidence currently available could limit the understanding of the main factors governing the trade-off between investment in medusa production and polyp-to-polyp propagation.

Many marine organisms employ some of the strategies discussed here, for example, the ubiquitous cysts and resting stages of plankton (Marcus 1996; Belmonte et al. 1997) and the dispersing larvae of many sessile organisms (Scheltema 1986). Scyphozoans and other jellyfish are unique in the diversity of life histories present within and across species and are now routinely reared in laboratory cultures (Purcell et al. 2012). They are thus highly suitable model systems for the study of life-history evolution in the marine environment and for testing theoretical ideas such as those present in this article. As a first attempt to analyze scyphozoan polyp reproduction in a systematic way, we have simplified the diversity of asexual reproductive modes to highlight their functionality instead of physiological detail and drawn inspiration from the theoretical literature on the evolution of reproductive strategies in plants, which present many similar aspects. We hope that our results can contribute to an enhanced understanding of the intricacies of reproductive modes in marine organisms in general and of scyphozoan jellyfish in particular.

\section{Acknowledgments}

We would like to thank the Max Planck Institute for the Physics of Complex Systems for generously hosting N.A.S.-M. and S.P. during the initial development of this study. In addition, the authors would like to extend their thanks to the associate editor, Dr. Robin E. Snyder, and two anonymous readers for three very thorough reviews and for their excellent suggestions, which greatly improved this article.

\section{Literature Cited}

Adler, L., and G. Jarms. 2009. New insights into reproductive traits of scyphozoans: special methods of propagation in Sanderia malayensis GOETTE, 1886 (Pelagiidae, Semaeostomeae) enable establishing a new classification of asexual reproduction in the class Scyphozoa. Marine Biology 156:1411-1420.

Arai, M. N. 2009. The potential importance of podocysts to the formation of scyphozoan blooms: a review. Hydrobiologia 616:241246.

Belmonte, G., A. Miglietta, F. Rubino, and F. Boero. 1997. Morphological convergence of resting stages of planktonic organisms: a review. Hydrobiologia 355:159-165.

Childs, D. Z., C. J. E. Metcalf, and M. Rees. 2010. Evolutionary bethedging in the real world: empirical evidence and challenges revealed by plants. Proceedings of the Roval Society B 277:30553064.

Clark, K. B. 1975. Nudibranch life cycles in the Northwest Atlantic and their relationship to the ecology of fouling communities. Helgoländer Wissenschaftliche Meeresuntersuchungen 69:28-69.

Cohen, D. 1966. Optimizing reproduction in a randomly varying environment. Journal of Theoretical Biology 12:119-129.

Cohen, D., and S. A. Levin. 1987. The interaction between dispersal and dormancy strategies in varying and heterogeneous environments. Pages 110-122 in E. Teramoto and M. Yumaguti, eds. Lecture notes in biomathematics 71: mathematical topics in population biology, morphogenesis and neurosciences. Springer, Berlin. 1991. Dispersal in patchy environments: the effects of temporal and spatial structure. Theoretical Population Biology 39:6399.

Comins, H. N., W. D. Hamilton, and R. M. May. 1980. Evolutionarily stable dispersal strategies. Journal of Theoretical Biology 82:205230.

Condon, R. H., W. M. Graham, C. M. Duarte, K. A. Pitt, C. H. Lucas, S. H. D. Haddock, K. R. Sutherland, et al. 2012. Questioning the rise of gelatinous zooplankton in the world's oceans. BioScience 62: 160-169.

Graham, W. M., S. Gelcich, K. L. Robinson, C. M. Duarte, L. Brotz, J. E. Purcell, L. P. Madin, et al. 2014. Linking human well-being and jellyfish: ecosystem services, impacts, and societal responses. Frontiers in Ecology and the Environment 12:515-523.

Hamner, W. M., and M. N. Dawson. 2009. A review and synthesis on the systematics and evolution of jellyfish blooms: advantageous aggregations and adaptive assemblages. Hydrobiologia 616:161191.

Harada, Y., and Y. Iwasa. 1994. Lattice population dynamics for plants with dispersing seeds and vegetative propagation. on Population Ecology 36:237-249.

Hidalgo, J., R. R. de Casas, and M. Á. Muñoz. 2016. Environmental unpredictability and inbreeding depression select for mixed dispersal syndromes. BMC Evolutionary Biology 16:1-12.

Hoover, R. A., R. Armour, I. Dow, and J. E. Purcell. 2012. Nudibranch predation and dietary preference for the polyps of Aurelia labiata (Cnidaria: Scyphozoa). Hydrobiologia 690:199-213. 
Imbert, E. 2002. Ecological consequences and ontogeny of seed heteromorphism. Perspectives in Plant Ecology, Evolution and Systematics 5:13-36.

Kobayashi, Y., and N. Yamamura. 2000. Evolution of seed dormancy due to sib competition: effect of dispersal and inbreeding. Iournal of Theoretical Biology 202:11-24.

Kussell, E., and S. Leibler. 2005. Phenotypic diversity, population growth, and information in fluctuating environments. Science 309: 2075-2078.

Levin, S. A., D. Cohen, and A. Hastings. 1984. Dispersal strategies in patchy environments. Theoretical Population Biology 26:165-191.

Lucas, C. H. 2001. Reproduction and life history strategies of the common jellyfish, Aurelia aurita, in relation to its ambient environment. Hydrobiologia 451:229-246.

Lucas, C. H., W. M. Graham, and C. Widmer. 2012. Jellyfish life histories: role of polyps in forming and maintaining scyphomedusa populations. Advances in Marine Biology 63:133-196.

Lucas, C. H., and J. A. Williams. 1994. Population dynamics of the scyphomedusa Aurelia aurita in Southampton Water. Journal of Plankton Research 16:879-895.

Marcus, N. H. 1996. Ecological and evolutionary significance of resting eggs in marine copepods: past, present, and future studies. Hydrobiologia 320:141-152.

Møller, L. F., and H. U. Riisgård. 2007. Impact of jellyfish and mussels on algal blooms caused by seasonal oxygen depletion and nutrient release from the sediment in a Danish fjord. Journal of Experimental Marine Biology and Ecology 351:92-105.

Purcell, J. E., D. Atienza, V. Fuentes, A. Olariaga, U. Tilves, C. Colahan, and J.-M. Gili. 2012. Temperature effects on asexual reproduction rates of scyphozoan species from the northwest Mediterranean Sea. Hydrobiologia 690:169-180.

Ronce, O., F. Perret, and I. Olivieri. 2000. Evolutionarily stable dispersal rates do not always increase with local extinction rates. American Naturalist 155:485-496.

Ruiz, J., L. Prieto, and D. Astorga. 2012. A model for temperature control of jellyfish (Cotylorhiza tuberculata) outbreaks: a causal analysis in a Mediterranean coastal lagoon. Ecological Modelling 233:59-69.
Scheltema, R. S. 1986. On dispersal and planktonic larvae of benthic invertebrates: an eclectic overview and summary of problems. Bulletin of Marine Science 39:290-322.

Schiariti, A., V. Melica, T. Kogovšek, and A. Malej. 2015. Densitydependent effects control the reproductive strategy and population growth of Aurelia aurita s.l. scyphistomae. Marine Biology 162:1665-1672.

Schiariti, A., A. C. Morandini, G. Jarms, R. von Glehn Paes, S. Franke, and H. Mianzan. 2014. Asexual reproduction strategies and blooming potential in Scyphozoa. Marine Ecology Progress Series 510:241-253.

Schnedler-Meyer, N. A., S. Pigolotti, and P. Mariani. 2018. Data from: Evolution of complex asexual reproductive strategies in jellyfish. American Naturalist, Dryad Digital Repository, https://doi.org /10.5061/dryad.506g4.

Snyder, R. E. 2006. Multiple risk reduction mechanisms: can dormancy substitute for dispersal? Ecology Letters 9:1106-1114.

Takao, M., H. Okawachi, and S.-I. Uye. 2014. Natural predators of polyps of Aurelia aurita s.l. (Cnidaria: Scyphozoa: Semaeostomeae) and their predation rates. Plankton and Benthos Research 9:105113.

Touchon, J. C., and K. M. Warkentin. 2008. Reproductive mode plasticity: aquatic and terrestrial oviposition in a treefrog. Proceedings of the National Academv of Sciences of the USA 105:74957499.

Vagelli, A. A. 2007. New observations on the asexual reproduction of Aurelia aurita (Cnidaria, Scyphozoa) with comments on its life cycle and adaptive significance. Invertebrate Zoology 4:111-127.

Venable, D. L., and J. S. Brown. 1988. The selective interactions of dispersal, dormancy, and seed size as adaptations for reducing risk in variable environments. American Naturalist 131:360-384.

Vitalis, R., F. Rousset, Y. Kobayashi, I. Olivieri, and S. Gandon. 2013. The joint evolution of dispersal and dormancy in a metapopulation with local extinctions and kin competition. Evolution 67: 1676-1691.
Associate Editor: Robin E. Snyder Editor: Alice A. Winn

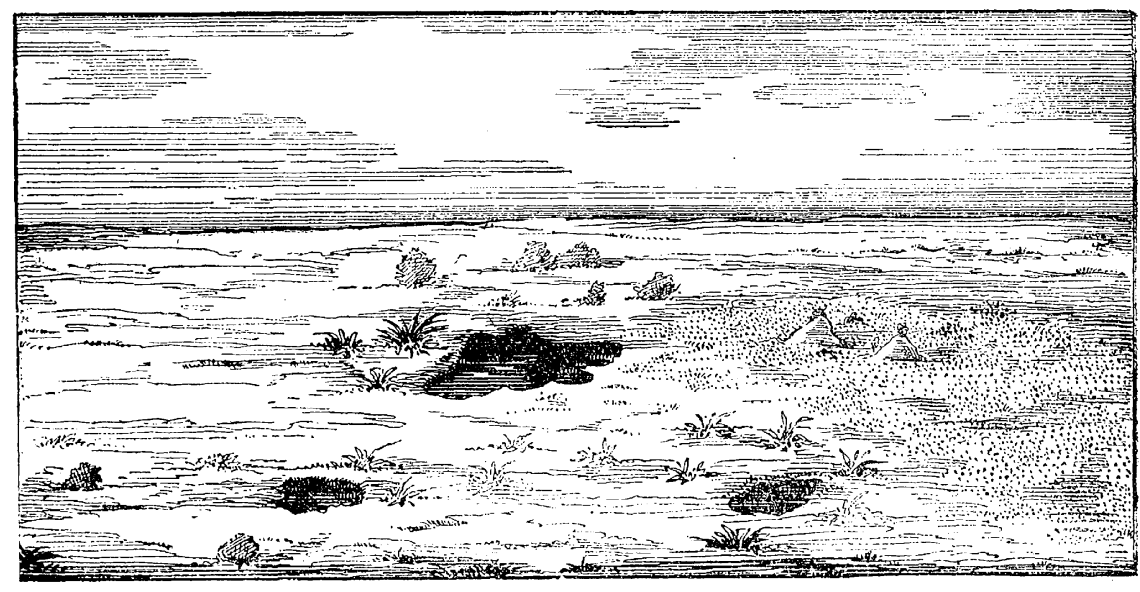

"To those who have experienced the pangs of thirst, while journeying over the desolate wastes that characterize this section, it will not be surprising that reminiscences of water should linger longest in the memory of the traveler. . . The springs in this portion of the Great Basin are few, and often far between. Their waters differ much in quantity, temperature and chemical composition." From "The Springs of Southern Nevada" by D. A. Lyle (The American Naturalist, 1878, 12:18-27). 